Z. Klin. Chem. Klin. Biochem.

11. Jg. 1973 , S. $31-34$

\title{
Bestimmung der Glucose nach der GOD-Perid1)-Methode mit dem Technicon SMA 6/60
}

\author{
Von J. SCHREIBER und R. LACHENICHT \\ Institut für Laboratoriumsdiagnostik der LVA Oberbayern im Zentralkrankenhaus Gauting (Leiter: MD Prof. Dr. K. F. \\ Petersen) und Boebringer Mannbeim GmbH, Biochemica Werk Tutzing
}

(Eingegangen am 21. März/5. Oktober 1972)

Es wird ein Verfahren zur Bestimmung der Glucose im Serum nach der GOD-Perid ${ }^{1}$ )-Methode mit dem Technicon-SMA 6/60 beschrieben. Präzision, Richtigkeit, Meßbereich und die Beeinflussung der Methode durch reduzicrende Substanzen werden angegeben und diskutiert.

\section{Determination of glucose by the GOD-Perid-method with the Technicon SMA 6/60}

A procedure, based on the GOD-Perid-Method is described for the determination of glucose in serum. The precision, accuracy, range of measurement, and interference by reducing substances are presented and discussed.

Da die enzymatischen Methoden zur Bestimmung der Glucose Vorteile bezüglich Präzision und Richtigkeit gegenüber den sogenannten Reduktionsmethoden bieten, haben wir die GOD-Perid'1)-Methode (1) auf den Technicon SMA 6/60 adaptiert. Die Verwendung von ABTS $^{2}$ ) (Ammoniumsalz der 2,2'-Azino-di-[3-äthylbenzthiazolinsulfonsäure-(6)]) als Redoxindikator, statt z. B. o-Dianisidin, führt zu einer wesentlichen Verbesserung der enzymatischen Glucose-Bestimmung mit Analysenautomaten (2).

\section{Methodik}

Prinzip

Nach erfolgter Blutentnahme läßt man das Blut etwa 30 min stehen und zentrifugiert. Das so gewonnene Serum wird in Probenbecher dekantiert und dem Gerät unverdünnt zugeführt, wo es mit einer Kupfer[II]-sulfat und Neocuproin enthaltenden Lösung vermischt wird. Störende reduzierende Substanzen, wie z. B. Harnsäure, werden im System durch Kupfer[II]-ionen oxidiert und die entstehenden Kupfer[I]-ionen durch Neocuproin komplex gebunden. Die Abtrennung von Eiweiß erfolgt durch Dialyse gegen Natriumchlorid-Lösung. Nach der Dialyse wird Enzym ${ }^{3}$ )-ABTS-Reagenz zugeführt und die Glucose wie folgt umgesetzt:

\section{Reaktionsgleichungen: \\ Glucose $+\mathrm{O}_{2}+\mathrm{H}_{2} \mathrm{O} \underset{\text { Glucoseoxidase }}{\longrightarrow}$ Gluconsäure $+\mathrm{H}_{2} \mathrm{O}_{2}$ $\mathrm{H}_{2} \mathrm{O}_{2}+$ ABTS $\stackrel{\text { Peroxidase }}{\longrightarrow}$ Farbstoff $+\mathrm{H}_{2} \mathrm{O}$.}

Der gebildete grüne Farbstoff wird bei 420 nm gemessen.

Reagenzien und Lösungen

Reagenzien

1. Natriumfluorid p. a. (Merck 6449)

2. Natriumchlorid p. a. (Merck 6404)

3. Kupfersulfat $5 \mathrm{H}_{2} \mathrm{O}$ p. a. (Merck 2790)

4. Neocuproinhydrochlorid, 2,9-Dimethyl-1,10-phenanthrolinhydrochlorid (Fluka 72090)

5. di-Natriumhydrogenphosphat wasserfrei LAB (Merck 6586)

6. Natrium-dihydrogenphosphat $\cdot \mathrm{H}_{2} \mathrm{O}$ p. a. (Merck 6346)

7. Glucoseoxidase (GOD II, Boehringer Manṇheim 15426)

8. Peroxidase (POD II, Boehringer Mannheim 15302)
9. ABTS (Boebringer Mannheim 15594)

10. Tween 20 (Firma Unfried)

11. Kontrollserum, z. B. Precinorm ${ }^{4}$ ) S (Boehringer Mannheim 15919)

12. SMA Reference Serum (Technicon T 03-0308-51).

\section{Lösungen}

Fluorid-Verdünnungsreagenz $\quad\left(0,6 \mathrm{mmol} / \mathrm{l} \quad \mathrm{CuSO}_{4} ; 0,6 \mathrm{mmol} / \mathrm{l}\right.$ Neocuproinhydrochlorid; $24 \mathrm{mmol} / \mathrm{l} \mathrm{NaF} ; 154 \mathrm{mmol} / \mathrm{l} \mathrm{NaCl}$ ). $0,600 \mathrm{~g}$ Kupfer[II]-sulfat $5 \mathrm{H}_{2} \mathrm{O}, 0,588 \mathrm{~g}$ Neocuproinhydrochlorid, $4 \mathrm{~g}$ Natriumfluorid und $36 \mathrm{~g}$ Natriumchlorid in 41 bidest. Wasser unter Zusatz von $1,0 \mathrm{ml}$ Tween 20 lösen oder Zusatzpackung für Analysenautomaten „Fluorid-Verdünnungsreagenz" (Boehringer Mannheim 15748) verwenden. Bei Raumtemperatur 2 Monate haltbar.

Natriumchlorid-Lösung (154 mmol/l).

$36 \mathrm{~g}$ Natriumchlorid in 41 bidest. Wasser unter Zusatz von $2,0 \mathrm{ml}$ Tween 20 lösen.

Enzym-ABTS-Reagenz (200 mmol/1 Phosphat-Puffer, $\mathrm{pH}=7,0$; $24 \mathrm{kU} / 1$ Glucoseoxidase; $2,0 \mathrm{kU} / 1$ Peroxidase; 2,0 g ABTS/1).

$19,0 \mathrm{~g}$ di-Natriumhydrogenphosphat (wasserfrei), 9,169 g Natriumdihydrogenphosphat $\cdot \mathrm{H}_{2} \mathrm{O}, 2,0 \mathrm{~g}$ ABTS, $24 \mathrm{kU}$ Glucoseoxidase und 2;0 kU Peroxidase oder Inhalt einer Flasche 1 der Packung für Analysenautomaten „Blutzucker GOD-PeridMethode" (Boehringer Mannheim 15703) in 11 bidest: Wasser lösen. In dunklex Flasche bei Raumtemperatur drei Tage, bei $+4^{\circ} \mathrm{C}$ sechs Wochen haltbar.

\section{Kalibrietung}

Der Glucose-Sollwert des Referenzstandards der Firma Technicon für das SMA-System wird nicht nach einer enzymatischen Methode ermittelt, sondern durch die Neocuproin-Methode.

Es ist daher notwendig, die Glucosekonzentration des Referenzstandards einer jeden Charge durch Eichung mit einem Kontroll-

1) GOD-Perid ist eingetragenes Warenzeichen der Boehringer Mannheim GmbH.

2) ABTS ist eingetragenes Warenzeichen der Boehringer Mannheim $\mathrm{GmbH}$.

3). Enzyme: Glucoseoxidase ( $\beta$-D-Glucose: oxygen oxidoreductase, EC 1.1.3.4); Pcroxidase (Donor: hydrogen-peroxide oxidoreductase, EC 1.11.1.7).

1) Precinorm $S$ ist eingetragenes Warenzeichen der Boehringer Mannheim GmbH. 


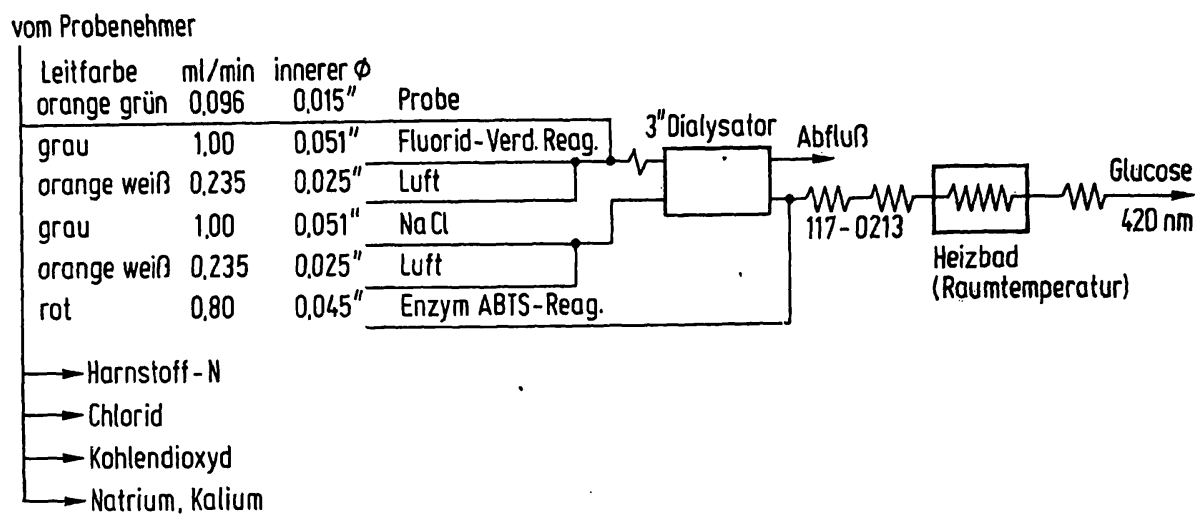

\author{
1 \\ Abb. 1
}

Fließdiagramm für die Bestimmung der Glucose nach der GOD-Perid-Methode mit dem Technicon SMA 6/60 serum zu korrigieren, dessen Glucosegehalt mittels der GODPerid-Methode enzymatisch ermittelt worden ist, wie z. B. mit Precinorm S.

\section{Ausführung}

Die analytischen Einschübe des SMA $6 / 60$ bestehen aus einem in sich geschlossenen System mit allen Vorrichtungen zum Mischen, Dialysieren und Inkubieren. Dieses System für die GlucoseBestimmung nach der Neocuproinmethode (3) wird weiter verwendet, jedoch geringfügig modifiziert. Durch Herausziehen des Steckers wird das Heizbad abgeschaltet und bei Raumtemperatur benutzt.

Das Fließdiagramm ist aus Abbildung 1 ersichtlich. Gegenüber der Neocuproin-Methode wird der Reagenz pumpende Schlauch auf $0,045^{\prime \prime}$ erweitert und ein Filter der Wellenlänge $420 \mathrm{~nm}$ statt $460 \mathrm{~nm}$ verwendet.

\section{Ergebnisse und Diskussion}

\section{Meßbereich}

Der Meßbereich erstreckt sich von $50-400 \mathrm{mg} / 100 \mathrm{ml}$ (3-22 mmol/l) Glucose. Bestimmungen von Standardlösungen mit 50,100,150, 200, 300 und $400 \mathrm{mg} / 100 \mathrm{ml}$ Glucose in Albuminlösung $\left.{ }^{5}\right)(60 \mathrm{~g} / \mathrm{l})$ ergeben eine gute Linearität, wie aus der Abbildung 2 ersichtlich ist.

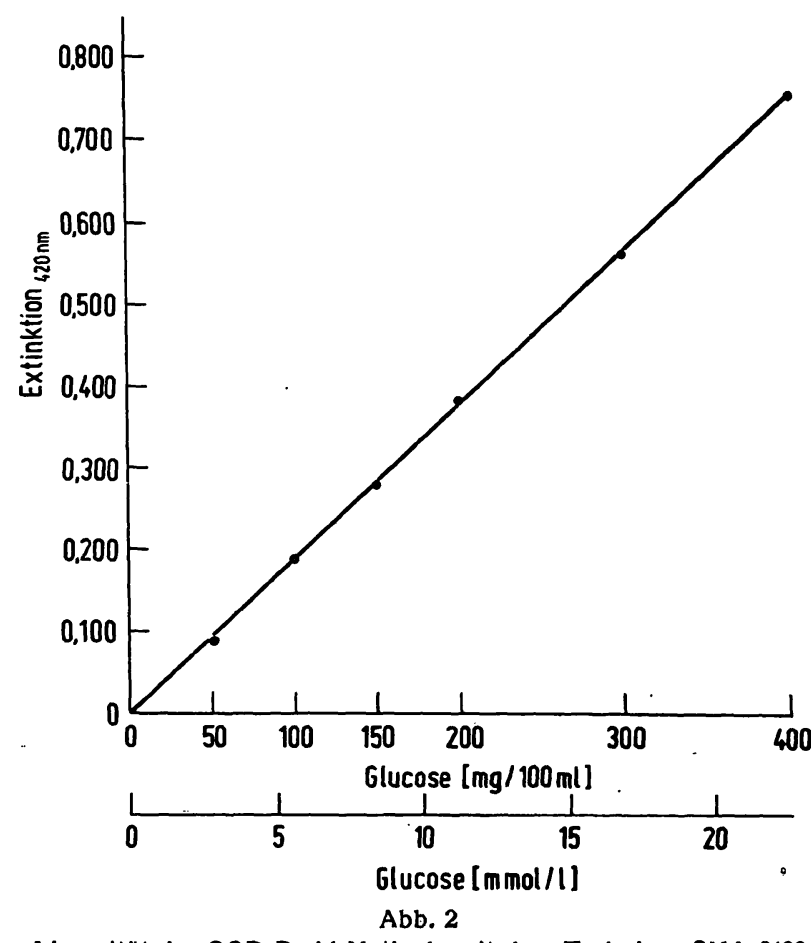

Linearität der GOD-Perid-Methode mit dem Technicon SMA 6/60

\section{Präzision}

Je 20 Proben eines Poolserums mit Glucosekonzentrationen im physiologischen und pathologischen Bereich wurden in der Serie untersucht. Die Mittelwerte $(\overline{\mathrm{x}})$ betrugen 63 bzw. $238 \mathrm{mg} / 100 \mathrm{ml}$ Glucose bei einer Streuung (s) von $0,7 \mathrm{bzw} .1,8 \mathrm{mg} / 100 \mathrm{ml}$. Daraus errechneten sich Variationskoeffizienten von $\pm 1,0 \mathrm{bzw}$. $\pm 0,7 \%$. Die Präzision vọ Tag zu Tag wurde durch Bestimmung eines Poolserums an 25 Tagen ermittelt. Aus einem Mittelwert ( $\overrightarrow{\mathbf{x}})$ von 95,8 mg $100 \mathrm{ml} /$ Glucose und einer Streuung (s) von $2,0 \mathrm{mg} / 100 \mathrm{ml}$ ergab sich ein Variationskoeffizient (VK von $\pm 2,1 \%$ ).

\section{Richtigkeit}

Durch 200 Vergleichsuntersuchungen zwischen der manuell durchgeführten Hexokinase-Methode ${ }^{6}$ (4) und der hier beschriebenen Methode wurde die Richtigkeit überprüft. Abbildung 3 gibt die Ergebnisse aus den Parallelbestimmungen wieder. In einem Bereich von

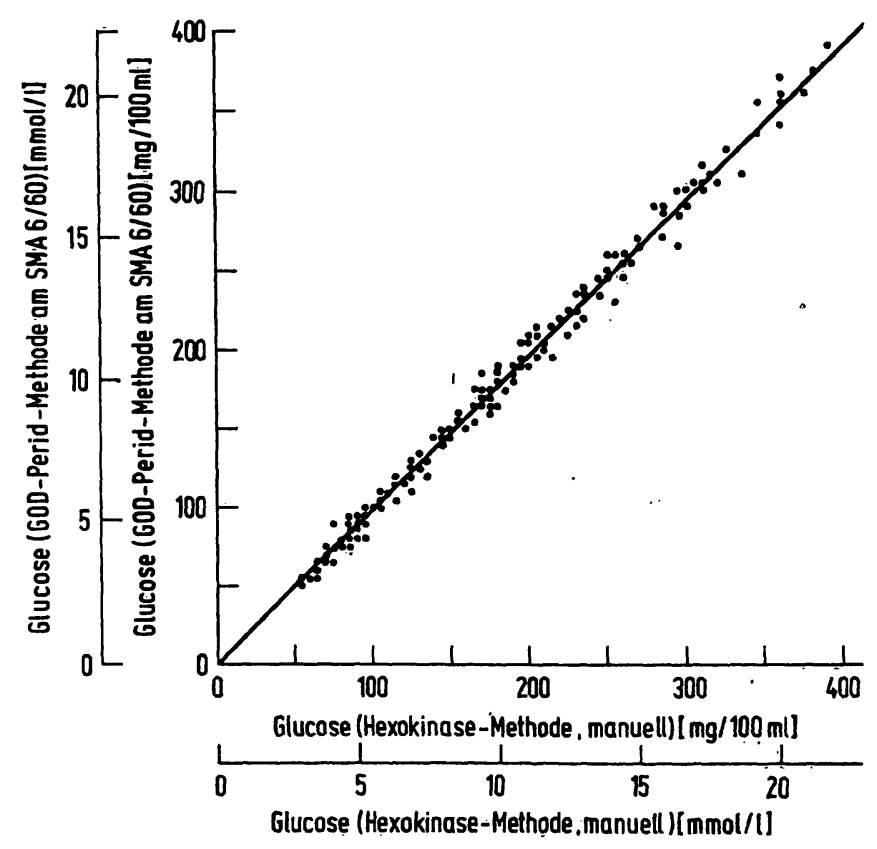
Abb. 3

Vergleichsuntersuchungen der GOD-Perid-Methode am Technicon SMA 6/60 und der manuell durchgeführten Glucose-Bestimmung nach der Hexokinase-Methode

5) Serumalbumin vom Rind, Behringwerke.

6) Test-Combination Glucose (Hexokinase-Methode) 15931, Boehringer Mannheim $\mathrm{GmbH}$. 
$51-442 \mathrm{mg} / 100 \mathrm{ml}$ ergab sich eine gute Übereinstimmung, wie aus den folgenden statistischen Daten hervorgeht. Es wurde eine Regressionsgerade von $y=3,89$ $+0,98 x$, eine Streuung $s_{y} \cdot x$ von $6,9 \mathrm{mg} / 100 \mathrm{ml}$ und ein Korrelationskoeffizient von 0,996 ermittelt.

Tabelle 1 zeigt die Ubereinstimmung der Glucose-Konzentration mit Sollwerten von verschiedenen Kontrollseren, die durch enzymatische Methoden bestimmt wurden. Dabei handelt es sich jeweils um Mittelwerte aus Doppelbestimmungen an zwei Tagen. Verschiedene Seren enthielten bis zu $10 \mathrm{mg} / 100 \mathrm{ml}$ Harnsäure $(594,8$ $\mu \mathrm{mol} / 1)$.

Einfluß durch Anwesenheit von reduzierenden Substanzen

Humanseren mit 94, 137, 187 und $211 \mathrm{mg} / 100 \mathrm{ml} \mathrm{Glu-}$ cose wurde Harnsäure bis zu $14 \mathrm{mg} / 100 \mathrm{ml}(832,7 \mu \mathrm{mol} / \mathrm{l})$ zugesetzt und diese mittels spektrophotometrischer manueller Uricase-Methode ${ }^{7}$ ) (5) bestimmt. Aus Abbildung 4 geht hervor, daß die beschriebene Methode nur bei sehr hohen Harnsäure-Konzentrationen geringfügig niedrigere Glucosewerte liefert. Geäußerte Bedenken gegen ABTS als Indikatorreagenz bezüglich Harnsäure-Interferenzen (6) sind durch dieses neue Verfahren aufgehoben.

Abbildung 4 zeigt außerdem, daß Ascorbinsäure bis zu $4 \mathrm{mg} / 100 \mathrm{ml}(227 \mu \mathrm{mol} / \mathrm{l})$ Serum keinen Einfluß auf die Methode hat. Jeder einzelnen Markierung des Diagramms liegen Doppelbestimmungen zugrunde. Wäßrigen Glucosestandard-Lösungen und Humanseren wurde Creatinin von $1-6 \mathrm{mg} / 100 \mathrm{ml}(90-540 \mu \mathrm{mol} / \mathrm{l}) \mathrm{zu}-$ gesetzt und die Glucosekonzentration am Technicon SMA 6/60 ermittelt (Doppelbestimmungen). Tabelle 2 verdeutlicht, $d a ß$ anwesendes Creatinin nicht stört.

Die Reagenzien sind in Wasser gut löslich. Durch die gute Stabilität erübrigt sich das tägliche Ansetzen der Lösungen. Eine nahezu gleichbleibende Farbe der 7) Test-Combination Harnsäure, 15986, Boehringer Mannheim GmbH.
Enzym-ABTS-Lösung sowie der hohe molare Extinktionskoeffizient des gebildeten Farbstoffes zeichnen die Methode besonders aus. Die Präzision und vor allem die gute Ubereinstimmung zur manuell durchgeführten Hexokinase-Methode sprechen für die Anwendung dieses enzymatischen Farbtestes am Technicon SMA $6 / 60$.

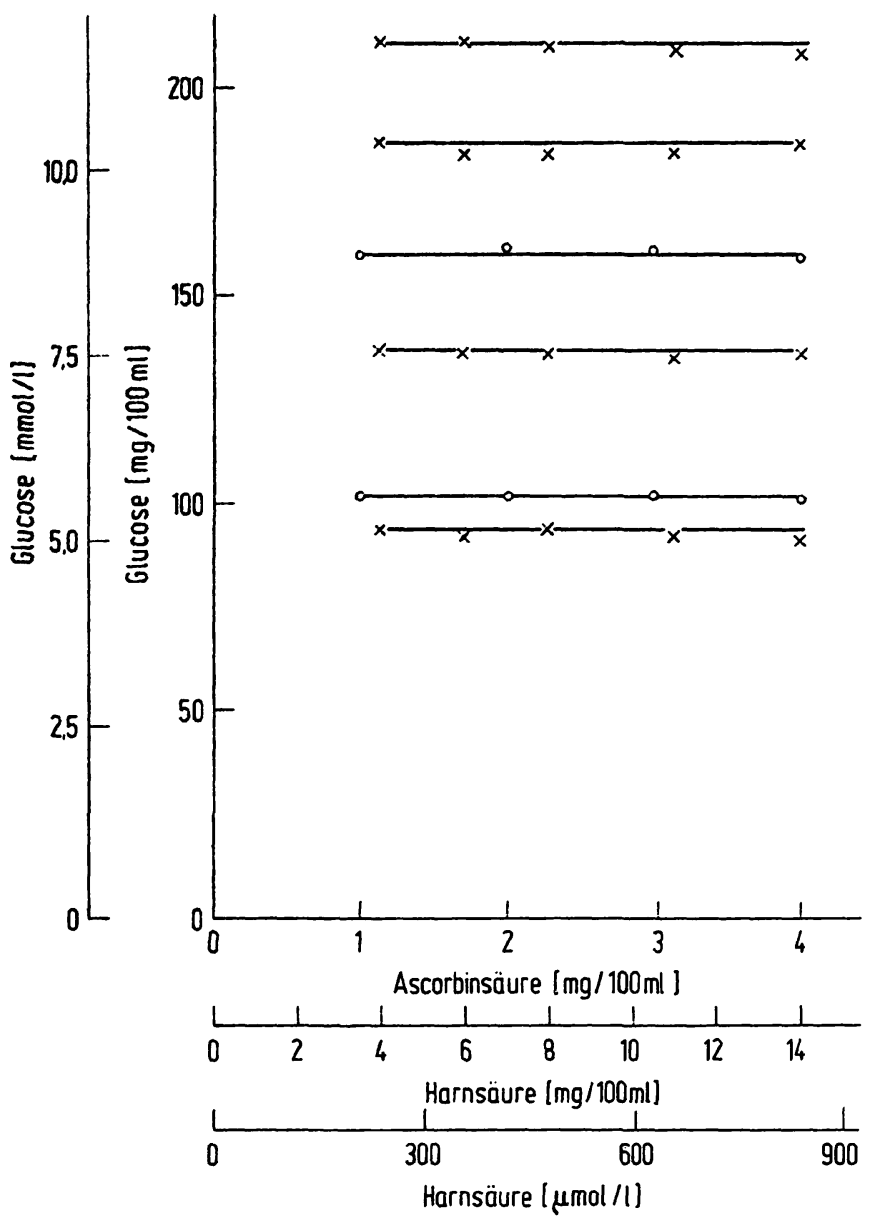

\section{Abb. 4}

Beinflussung der GOD-Perid-Methode am Technicon SMA 6/60 durch Anwesenheit von $4-11 \mathrm{mg} / 100 \mathrm{ml}(240-660 \mu \mathrm{mol} / \mathrm{l})$ Harnsäure, gekennzeichnet durch Kreuze und $1-4 \mathrm{mg} / 100 \mathrm{ml}$ Ascorbinsäure,
durch Kreise dargestellt

Tab. 1

Gegenüberstellung von Sollwerten verschiedener Kontrollseren mit gefundenen Werten der GOD-Perid-Methode mit dem Technicon SMA 6/60

\begin{tabular}{|c|c|c|c|}
\hline \multirow[t]{2}{*}{ Kontrollserum } & \multicolumn{2}{|c|}{$\begin{array}{l}\text { Sollwert Glucose } \\
\text { manuelle Methoden } \\
{[\mathrm{mg} / 100 \mathrm{ml}]}\end{array}$} & \multirow{3}{*}{$\begin{array}{c}\text { Gefundener Wert } \\
\text { Glucose } \\
\text { GOD-Perid-Methode } \\
\text { SMA } 6 / 60 \\
{[\mathrm{mg} / 100 \mathrm{ml}]}\end{array}$} \\
\hline & Glucoseoxidase & Hexokinase & \\
\hline Humanserum (Behringwerke) & 83 & 86 & \\
\hline Seronorm (Nyegaard, Oslo) & 117 & & 116 \\
\hline Lab-trol $\left.{ }^{9}\right)($ Dade) $)$ & 99 & 95 & 105 \\
\hline Patho-trole) (Dade)") & 275 & 257 & 278 \\
\hline Versatol") (General Diagnostics) & 85 & & 80 \\
\hline Versatol ${ }^{\circ}$ ) A (General Diagnostics) & 204 & 14 & 202 \\
\hline Versatol () A Alt. (General Diagnostics) & 307 & $\mathbf{n}$ & 308 \\
\hline Hyland Normal (Hyland Travenol) & 93 & 91 & 90 \\
\hline Hyland Abnormal (Hyland Travenol) & 179 & 1 & 176 \\
\hline Precinorm's (Boehringer Mannheim) & 107 & 106 & 106 \\
\hline
\end{tabular}

8) Lab-trol, Patho-trol und Dade sind eingetragene Warenzeichen der Dade Division American Hospital Supply Corporation Miami, Florida.

9) Versatol ist eingetragenes Warenzeichen der Firma General Diagnostics, Division Warner-Chilcott Laboratories, Morris-Plains N. J. 
Tab. 2

Beeinflussung der GOD-Perid-Methode durch Anwesenheit von $1-6 \mathrm{mg} / 100 \mathrm{ml}(90-540 \mu \mathrm{mol} / \mathrm{l})$ Creatinin

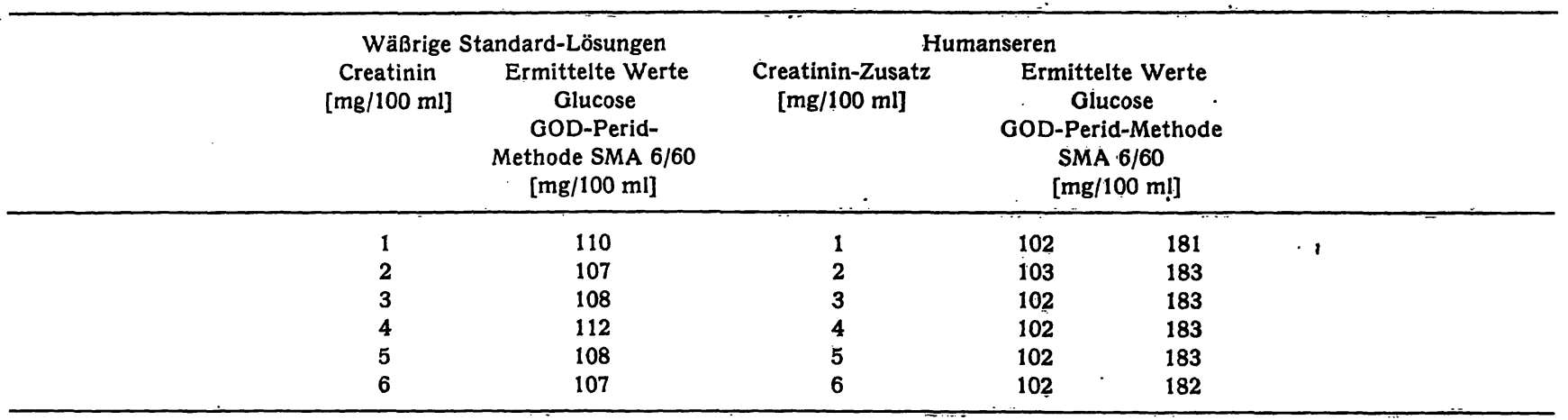

\section{Literatur}

1. Werner, W., Rey, H.-G. \& Wielinger, H. (1970) Z. Anal. Chem. 252, 224-228. - 2. Kahle, K., Werss, L., Klarwein, M. \& Wieland, O. (1970) Z. Anal. Chem. 252, 228-231. - 3. Technicon Methodologie AA II-2. - 4. Schmid, F. H. (1961) Klin.
Wochenschr. 39, 1244-1247. - 5. BERNT, E. \& Bergmeyer, H. U. (1970) in Methoden der enzymatischen Analyse, S. 1885 -1887 (H. U. Bergmeyer, ed.) 2. Aufl., Weinheim. -6. Eberhardt, D., Decker, S. \& KlingmülleR, V. (1971) diese Z. 9, 362-363.

R. Lachenicht, Bio. Ausl. Boehringer Mannheim GmbH 6800 Mannheim 31

Postfach 51 